\title{
Perfil epidemiológico oral y necesidad de tratamiento odontológico de pacientes VIH/SIDA
}

\author{
María del Carmen Jiménez Malagón ${ }^{\mathrm{a}}$, Luis Fang ${ }^{\mathrm{b}}$, Antonio Diaz Caballero
}

a Odontóloga. Especialista en Estomatología y Cirugía Oral. Universidad de Cartagena. Profesor Titular Facultad de Odontología Universidad de Cartagena.

' Odontólogo. Magister en Inmunología. Universidad de Cartagena.

c Odontólogo Universidad de Cartagena. Especialista en Periodoncia Universidad Javeriana. Magister en Educación Universidad del Norte. Estudiante de Doctorado en Ciencias Biomédicas Universidad de Cartagena. Docente titular Universidad de Cartagena. Director Grupo de investigaciones GITOUC.

Correspondencia: Luis Fang Mercado, Facultad de Odontología Universidad de Cartagena, Campus de la Salud, Barrio Zaragocilla, Cartagena, Bolivar, Colombia, Sur América.

Teléfono $057+5+6698172$

Ext. 110 Fax 057+5+6698173

Ext. 124. Correo electrónico: luifang87@gmail.com y mcjimenezm@gmail.com

Recibido el 17 de febrero de 2012.

Aceptado para su publicación el 22 de marzo de 2012.

\section{RESUMEN}

Objetivo. Describir el perfil epidemiológico oral y las necesidades de tratamiento odontológico en pacientes VIH/SIDA atendidos en una institución.

Diseño. Estudio descriptivo transversal.

Emplazamiento. Facultad de Odontología Universidad de Cartagena.

Participantes. Pacientes con diagnóstico de VIH/SIDA y bajo tratamiento antirretroviral.

Mediciones principales. Se evaluaron variables sociodemográficas, estado de Higiene Oral, historia de Caries, Enfermedad Periodontal, manifestaciones orales asociadas a VIH/SIDA, necesidad de tratamiento odontológico.

Resultados: De los 53 sujetos del estudio un $59,9 \%$ presentaron higiene oral regular, un $66 \%$ mostró cálculo supragingival y un 15,1\% sangrado gingival. La Se observó caries en el 92,5\% de los participantes y ausencia de dientes en el 73,6\%. La prevalencia de manifestaciones orales de $\mathrm{VIH} / \mathrm{SIDA}$ fue de un $45,3 \%$, destacando las de origen infeccioso con un $28,3 \%$. La presencia de lesiones no asociadas a $\mathrm{VIH}$ fue del $81,1 \%$, sobresaliendo con un $28,3 \%$ las pigmentaciones orales asociadas a medicamentos antirretrovirales. El 100\% de los sujetos precisó asistencia odontológica, necesitando 4 tipos de tratamiento diferentes el $60,4 \%$ de ellos. Sobresale la promoción y prevención en un $98.1 \%$ de los casos, seguida por la eliminación de cálculo en el $73,6 \%$ y la necesidad protésica en el $81,2 \%$ de los individuos.

Conclusión: Se hace evidente la falta de atención y la necesidad de tratamiento de esta población. La cual presenta un estado de salud oral regular, afirmando el derecho a la atención integral que este tipo de pacientes merece.

Palabras clave. SIDA, VIH, Examen oral, Índice de Higiene Oral, Atención Odontológica Integral.

\section{ABSTRACT}

Oral epidemiological profile and the need for dental treatment for HIV/AIDS patients

Objective. To describe the oral epidemiological profile and the needs for dental treatment for HIV/AIDS patients in an institution.

Design. Cross sectional study.

Location. University of Cartagena Faculty of Odontology

Participants. Patients diagnosed with HIV/AIDS undergoing antiretroviral treatment.

Main Measures. Socio-demographic variables, oral hygiene condition, history of tooth decay, periodontal disease, oral signs associated with HIV/AIDS, need for dental treatment were evaluated.

Results: Out of 53 study subjects, $59.9 \%$ showed mediocre oral hygiene, $66 \%$ supragingival calculus and $15.1 \%$, gingival bleeding. We observed tooth decay in $92.5 \%$ of the participants and absence of teeth in $73.6 \%$. The prevalence of oral signs of HIV/AIDS was $45.3 \%$, with emphasis on a $28.3 \%$ of an infectious origin. The presence of lesions not associated with HIV was $81.1 \%$, and a significant $28.3 \%$ with oral pigmentations associated with antiretroviral medication. $100 \%$ of the subjects required dental care, with 4 different types being necessary for $60.4 \%$ of them. Promotion and prevention were significant in $98.1 \%$ of cases, followed by removal of calculus in $73.6 \%$ y the need for prosthesis in $81.2 \%$ of individuals.

Conclusion: Lack of attention and the need for treatment is evident in these members of the population, who show mediocre oral health, strengthening the case for the comprehensive care this type of patient deserves.

Key words. AIDS, HIV, Diagnosis oral, Oral Hygiene Index, Comprehensive Dental Care. 


\section{INTRODUCCIÓN}

El sida es una enfermedad viral de carácter epidémico que ocasiona inmunosupresión profunda acompañada de infecciones por gérmenes oportunistas, neoplasias secundarias y manifestaciones neurológicas. La situación global de la epidemia de SIDA no es tranquilizadora para nadie, en el mundo se sobrepasa la cifra de 33,3 millones de personas infectadas. En Colombia actualmente según reportes de ONUSIDA la prevalencia de VIH/SIDA llega a los 109.000 infectados $^{1}$. En Cartagena de Indias, una ciudad turística de la costa norte colombiana, los reportes del año 2008 mostraron que el $67 \%$ de los casos correspondió a hombres y el $33 \%$ a mujeres, evidenciando un descenso en la proporción del género femenino. Sin embargo, siguen aumentado los casos en esta ciudad².

Al analizar el contexto socio-demográfico y cultural en el que se desarrollan los enfermos de VIH/SIDA se evidencia la estrecha relación que posee con su estado de salud general y, a la vez, con su salud oral. Nittayananta $\mathrm{W}$ et al. ${ }^{3}$, Dray-Spira $\mathrm{R}$ et al. ${ }^{4} \mathrm{y}$ Zungu-Dirwayi $\mathrm{N}$ et $\mathrm{al}^{5}$. Observaron una marcada relación entre las características socio-demográficas de los pacientes con VIH/SIDA y el estado de salud general.

En este mismo sentido, Santo A et al. ${ }^{6}$ mostraron que los pacientes con VIH/SIDA se consideran un grupo seriamente necesitado de los servicios odontológicos. Desde esta perspectiva este grupo poblacional está influido por factores sociodemográficos y culturales que repercuten en su dinámica poblacional, como fue reportado en Colombia por Pinzón E et al. ${ }^{7}$, en Venezuela por Navas R et al. ${ }^{8}$, en Brasil por Noce $\mathrm{C}$ et al. ${ }^{9}$ y México por Valdespino $\mathrm{JL}$ et al. ${ }^{10}$. Por este motivo, para poder brindar una atención integral a estos pacientes es necesario estudiar las características de cada población y diseñar un plan de tratamiento acorde a sus necesidades básicas como lo realizó Lemos $\mathrm{S}$ et al. ${ }^{11}$, Santana $Y$ et al. ${ }^{12}$ y Lambertini $A$ et al ${ }^{13}$.

Los estudios que relacionen el estado de salud oral con variables poblacionales y conductuales permiten obtener un enfoque más completo de las necesidades propias de los pacientes VIH positivos, para el mejoramiento de la calidad de vida de cada población.

Por todo lo referido, el objetivo de esta investigación consistió en describir el perfil epidemiológico oral en pacientes VIH/SIDA y necesidades de tratamiento odontológico.

\section{MATERIAL Y MÉTODOS}

Se realizó un estudio descriptivo transversal en una institución prestadora de salud de Cartagena de Indias que implementa un programa para la atención de población VIH/SIDA. La población de estudio estuvo constituida por 194 pacientes afiliados a esta institución. El tamaño de la muestra fue de 53 sujetos y se calculó a través de la formula estadística para población finita, considerando una prevalencia esperada del fenómeno de $95 \%$, un nivel de confianza del $95 \%$ y una precisión de $\pm 5 \%$. Se estableció un muestreo probabilístico con el método aleatorio simple, a partir de una lista completa de la población en la cual se le asignó un código a cada individuo y, finalmente, mediante una tabla de números aleatorios en Excel se seleccionaron los sujetos que formaron parte de la muestra.

Previamente a la selección se consideraron los siguientes criterios: ser usuarios de la institución prestadora de salud, diagnosticados con VIH/SIDA y bajo tratamiento antirretroviral. No se incluyeron aquellos que sufrieran de otras enfermedades sistémicas que pudieran presentar manifestaciones orales (Diabetes, Hipertensión, leucemia, entre otras), mujeres en estado gestante, consumidor de cigarrillo o alcohol.

Para la recogida de la información se diseñó un instrumento específico y se realizó entrevista y examen clínico. Se incluyó información de los tres grupos variables principales del estudio (sociodemográficas, estado de salud oral y necesidades de tratamiento).

De forma previa a la recogida de datos se informó a los participantes a través de un diálogo directo, donde se explicaron los objetivos de la investigación, los procedimientos a realizar, beneficios y riesgos a que estarían expuestos, garantizando la completa confidencialidad y privacidad de sus datos al momento de su socialización. Seguido a esto, se procedió a encuestar a los individuos con interrogantes relacionados a características sociodemográficas como: edad, sexo, nivel educativo, ocupación, estado conyugal y estrato socioeconómico.

El examen clínico intraoral fue realizado por un experto. Bajo las condiciones de protección y bioseguridad adecuadas se evaluaron: estado de higiene oral, por medio del índice de higiene oral simplificado (IHO-S), estado periodontal a través del Índice Comunitario Periodontal de Necesidades de Tratamiento (ICPNT) y la presencia de dientes ca- 
riados, perdidos y obturados. También se evaluó la presencia de manifestaciones orales propias de VIH/SIDA clasificadas según etiología ${ }^{14}$, lesiones orales de tejidos blandos no asociadas a VIH/SIDA y su respectiva localización.

A partir del examen clínico se establecieron las necesidades de tratamientos odontológicos en: promoción y prevención, periodoncia (raspado y alisado radicular y cirugía periodontal), cirugía (exodoncia y cirugías complejas), rehabilitación oral (corona individual, prótesis fija, prótesis parcial removible, prótesis total, operatorias), endodoncia (tratamiento de conductos radiculares) y ortodoncia.

Por otra parte, este estudio fue avalado por el comité de ética institucional y catalogado con riesgo mínimo a partir de las consideraciones emanadas por la resolución 008430 de 1993 del ministerio de protección social colombiano. Además todos los participantes firmaron consentimiento informado.

Para garantizar la calidad de los datos, se supervisó el diligenciamiento de las encuestas, se revisaron las distribuciones de frecuencia y tablas simples por cada una de las variables a fin de identificar códigos erróneos e información inconsistente. Se realizó un análisis univariado utilizando tablas de frecuencia y posteriormente análisis bivariado según sus características, tablas de contingencia, usando pruebas de ji-cuadrado de Pearson, corrección de yates y test exacto de Fisher, considerando un nivel de significación inferior a 0.05. La información recolectada se incluyó en una base de datos diseñada en Excel 2007 y para su análisis fue copiada al software estadístico SPSS STATISTICS v.17.0.

\section{RESULTADOS}

Se incluyeron en el estudio un total de 53 pacientes con VIH/SIDA, con una edad media de 36 años $\pm 13.3 \mathrm{DE}$. Los grupos etarios con mayor número de sujetos fueron el de 30 a 39 años y el de 40 a 49 años con un $32,1 \%$ para cada uno. El mayor porcentaje de los participantes pertenecen a la población económicamente activa, destacándose los que realizaban un trabajo independiente $(47,2 \%)$. Un $49,1 \%$ habían cursado estudios hasta primaria y la mayoría de la población estudiada era soltera $(69,8 \%)$. (Tabla 1).

El 59,9\% de los individuos evaluados presentó una higiene oral calificada como regular, presentando cálculo supragingival el $66 \%$ de ellos y sangrado gingival el $15,1 \%$ en los órganos dentarios evaluados. En cuanto a las lesiones cariosas, el 92,5\% de los participantes registraron presencia de caries, con una media de lesiones por individuo de 6,2 \pm $4,5 \mathrm{DE}$. El $73,6 \%$ de los participantes tienen ausencia de órganos dentarios por causa de caries, con un promedio de dientes perdidos por paciente de 5,5 \pm 6,9 DE (Tabla 2).

En cuanto a las manifestaciones orales asociadas a $\mathrm{VIH} / \mathrm{SIDA}$ la prevalencia general fue del $45,3 \%$. Atendiendo a la etiología de estas lesiones, destacaron en primer lugar las patologías de origen infeccioso con un $28,3 \%$, observando eritema gingival lineal en un $15,1 \%$ delosincluidos en el grupo de manifestaciones de origen bacteriano, mientras que en el grupo de las manifestaciones micóticas mostraron candidiasis eritematosa un $3,8 \%$. En segundo lugar se registraron las alteraciones de las glándulas salivales, representando en este caso la Xerostomía un 11,3\%. En tercer lugar figuraron las manifestaciones idiopáticas con un 5,7\% (Tabla 3).

Con relación a las lesiones orales de tejidos blandos no asociadas a $\mathrm{VIH}$, la prevalencia general fue del $81,1 \%$, destacando en este grupo las pigmentaciones orales, posiblemente asociadas con la ingesta de medicamentos antirretrovirales, con un $28,3 \%$. Las excrecencias óseas como Torus y Exostosis se manifestaron en el $17 \%$ de los individuos. La lengua saburral se registró en un 9,4\% y la queilitis exfoliativa en un $7,5 \%$. En el momento del examen clínico el 50,9\% presentaron entre 1 y 2 lesiones orales, y sólo una persona presentó cinco lesiones simultáneamente, independientemente de su etiología (Tabla 3).

Dentro de las necesidades de tratamiento odontológico, la totalidad de los individuos necesitaron atención odontológica. Un 60,4\% necesitó 4 tipos de tratamientos diferentes. Sobresale el porcentaje que precisó promoción y prevención con un $98,1 \%$ de los casos, al igual que el raspaje con un $73,6 \%$. La necesidad protésica se hace evidente en el $81,2 \%$ de los individuos (Tabla 4).

Al relacionar el análisis bivariado entre la presencia de cada patología con la edad, el sexo y el tiempo de diagnóstico no se observó significación estadística en ningún caso.

\section{DISCUSIÓN}

El estado de la salud oral de un individuo, es el eje principal para la toma de decisiones de los múlti- 


\begin{tabular}{|c|c|c|}
\hline VARIABLES & $\begin{array}{l}\text { FRECUENCIA } \\
\qquad(N=53)\end{array}$ & $\begin{array}{c}\text { PORCENTAJE } \\
\%\end{array}$ \\
\hline \multicolumn{3}{|l|}{ SEXO } \\
\hline - $\quad$ Femenino & 16 & 30,2 \\
\hline - $\quad$ Masculino & 37 & 69,8 \\
\hline \multicolumn{3}{|l|}{ EDAD (AÑOS) } \\
\hline$-<20$ & 5 & 9,4 \\
\hline$-\quad 20-29$ & 9 & 17 \\
\hline$-\quad 30-39$ & 17 & 32,1 \\
\hline$-\quad 40-49$ & 17 & 32,1 \\
\hline$-\quad>50$ & 5 & 9,4 \\
\hline \multicolumn{3}{|l|}{ ESTRATO SOCIAL } \\
\hline - Bajo & 48 & 90,6 \\
\hline - $\quad$ Medio & 5 & 9,4 \\
\hline - $\quad$ Alto & 0 & 0 \\
\hline \multicolumn{3}{|l|}{ ESTADO CIVIL } \\
\hline - Casado & 3 & 5,7 \\
\hline - Divorciado & 8 & 15,1 \\
\hline - Soltero & 37 & 69,8 \\
\hline - Unión libre & 5 & 9,4 \\
\hline \multicolumn{3}{|l|}{ ESCOLARIDAD } \\
\hline - Analfabeta & 1 & 1,9 \\
\hline - Primaria & 26 & 49,1 \\
\hline - Secundaria & 21 & 39,6 \\
\hline - Profesional & 5 & 9,4 \\
\hline \multicolumn{3}{|l|}{ OCUPACIÓN } \\
\hline - Independiente & 25 & 47,2 \\
\hline - $\quad$ Ama de casa & 14 & 26,4 \\
\hline - $\quad$ Desempleado & 8 & 15,1 \\
\hline - Patrón & 3 & 5,7 \\
\hline - Empleado & 3 & 5,7 \\
\hline
\end{tabular}

Tabla 1. Características Socio-demográficas en pacientes $\mathrm{VIH} /$ SIDA.

\begin{tabular}{l|cc}
\hline & $\begin{array}{c}\text { FRECUENCIA } \\
(\mathbf{N}=\mathbf{5 3})\end{array}$ & $\begin{array}{c}\text { PORCENTAJE } \\
\%\end{array}$ \\
\hline Estado de Higiene Oral & & \\
$-\quad$ Malo & 12 & 22,6 \\
$-\quad$ Regular & 27 & 50,9 \\
$-\quad$ Ótimo & 14 & 26,4 \\
Dientes cariados & 49 & 92,5 \\
Dientes Perdidos & 39 & 73,6 \\
Dientes Obturados & 30 & 37,7 \\
Calculo dental & 35 & 66,0 \\
Sangrado Gingival & 8 & 15,1 \\
\hline
\end{tabular}

Tabla 2. Estado de Salud Oral en pacientes VIH/SIDA. 


\begin{tabular}{l|cc}
\hline & $\begin{array}{c}\text { FRECUENCIA } \\
\text { (N=53) }\end{array}$ & $\begin{array}{c}\text { PORCENTAJE } \\
\%\end{array}$ \\
\hline Asociadas a VIH/SIDA según Etiología & & 28,3 \\
Infecciosa & 15 & 5,7 \\
Idiopática & 3 & 11,3 \\
Glandular & 6 & 0,0 \\
Neoplásicas & 0 & \\
No Asociadas a VIH/SIDA & & 28,3 \\
Pigmentaciones asociadas a la terapia antirretroviral & 15 & 17,0 \\
Torus y Exostosis & 9 & 9,4 \\
Lengua Saburral & 5 & 7,5 \\
Queilitis Exfoliativa & 4 & 5,7 \\
Estomatitis Subprotesica & 3 & 5,7 \\
Gránulos de Fordyce & 3 & 3,8 \\
Proceso Fistuloso & 2 & 1,9 \\
Leucoplasia & 1 & 1,9 \\
Pericoronitis & 1 & \\
\hline
\end{tabular}

Tabla 3. Lesiones Orales en pacientes VIH/SIDA.

\begin{tabular}{l|cc}
\hline & $\begin{array}{c}\text { FRECUENCIA } \\
(\mathbf{N}=\mathbf{5 3})\end{array}$ & $\begin{array}{c}\text { PORCENTAJE } \\
\%\end{array}$ \\
\hline Promoción y Prevención & 52 & 98,1 \\
Raspaje & 39 & 73,6 \\
Cirugía Periodontal & 2 & 3,8 \\
Exodoncia & 19 & 35,8 \\
Cirugía Compleja & 4 & 7,5 \\
Operatoria & 43 & 81,1 \\
Rehabilitación Oral & & \\
$\quad-\quad$ Prótesis Fija & 14 & 26,4 \\
$\quad-\quad$ Prótesis Removible & 23 & 43,4 \\
$\quad$ Prótesis Total & 5 & 9,4 \\
Endodoncia & 3 & 5,7 \\
Ortodoncia & 3 & 5,7 \\
\hline
\end{tabular}

Tabla 4. Necesidad de Tratamiento odontológico en pacientes VIH/SIDA.

ples tratamientos odontológicos que este requiere. En este sentido, en el presente estudio se encontró higiene oral regular entre los participantes, presencia de caries, órganos dentarios ausentes, cálculos y sangrado gingival, observando resultados similares a los presentados por Ribeiro et al. ${ }^{15}$ y Santana ${ }^{12}$, quienes igualmente mostraron altos porcentajes de individuos con estas afecciones. Para el Estudio Nacional de Salud Bucal ENSABIII ${ }^{16}$ de 1998 realizado en Colombia en personas sin enfermedad infectocontagiosa, el nivel de higiene oral regular fue de $39,4 \%$. En este estudio la prevalencia de caries fue de $65,3 \%$ y la presencia de cálculo fue del $53,3 \%$. Los resultados de nuestro 
estudio muestran porcentajes muy superiores a los referidos con un $92,5 \%$ de sujetos con caries, un $59,9 \%$ con higiene oral regular y un $66 \%$ con cálculo supragingival. Estos resultados podrán indicar la posible relación entre la presencia de esta patología infecciosa y el estado de salud oral de los que la padecen. Se ha afirmado que el estado de salud oral de este tipo de pacientes podría considerarse además un factor de riesgo para su bienestar ${ }^{17}$.

En nuestro estudio, la prevalencia general de manifestaciones orales asociadas a VIH/SIDA fue del 45,3. Estos resultado son similares a los de Ramírez Amador et al. ${ }^{18}$, que mostraron un prevalencia de $48,2 \%$ y $44,2 \%$ de manifestaciones asociadas y fuertemente asociadas, respectivamente, en una institución clínica. La prevalencia observada por Noce et al. ${ }^{9}$ fue de $35,8 \%$, mientras que en el estudio realizado en Colombia por Pinzon ${ }^{7}$ fue muy superior, alcanzando un $77,1 \%$. Las diferencias en la prevalencias observadas pueden estar en relación con las diferentes características y particularidades de cada población estudiada, con el contexto sociocultural en el que se desarrollaron las investigaciones y, posiblemente, con los criterios de selección de cada estudio. Además, el tipo de clasificación de manifestaciones orales utilizado constituye un factor determinante para obtener la prevalencia de la misma, aunque en la actualidad se ha observado una tendencia a disminuir, debido a la aplicación de terapia antirretroviral altamente $\operatorname{activa}^{17,19}$.

En este estudio se escogió la clasificación de las manifestaciones orales $\mathrm{VIH} / \mathrm{SIDA}$ según agente etiológico, publicada por Shiboski et al. ${ }^{14}$ por intermedio del Oral HIV/AIDS Research Alliance (OHARA), con la finalidad de implementar una nueva clasificación más acorde a las lesiones orales con mayor incidencia y prevalencia en los últimos años 7.9.17.18.20.21. Es lógico pensar que siendo esta clasificación relativamente nueva no se encuentre literatura que implemente esta clasificación ${ }^{14}$. Por este motivo no es posible comparar los resultados referidos a este apartado con otros estudios, sin embargo sí se discutieron datos individuales de algunas manifestaciones presentes.

La presencia de un $15,1 \%$ de participantes con eritema gingival es superior a la referida en algunos estudios con una $2,7 \%{ }^{18}$ y un $7,1 \%{ }^{9}$, aunque otros autores mostraron una prevalencia más elevada ${ }^{7}$. La candidiasis eritematosa afectó al $3,8 \%$ de los casos, que es un porcentaje muy inferior al registrado en la literatura, refiriendo cifras del $12,2 \%{ }^{9}$ y $17,5 \%{ }^{18}$. Respecto a la estomatitis aftosa recurrente nuestros resultados fueron similares a los de otros estudios que mostraron cifras del $3,5 \%{ }^{9}$ y $4,9 \%{ }^{18}$.

Con relación a la necesidad de tratamiento, se hace evidente la necesidad protésica en un $81,2 \%$ de los participantes. Esta necesidad fue superior a la referida por Lambertini ${ }^{13}$ y por Santana $Y^{12}(73,6 \%$ y $66,7 \%$, respectivamente) y a la mostrada en el Estudio Nacional De Salud Bucal-ENSAB III ${ }^{16}$ que fue del $68,9 \%$. La necesidad de procedimientos operatorios dentales fue del $81,1 \%$, que es muy superior a los datos publicados ${ }^{12}$. Estos resultados resaltan la necesidad de atención odontológica de estos pacientes ${ }^{12.13}$.

Los resultados observados permiten elaborar un plan de atención odontológico completo, en el cual se pueden identificar las principales afecciones que presentan los pacientes VIH/SIDA y planear el tratamiento ideal acorde a las necesidades básicas. Es muy importante para las entidades reguladoras registrar cada una de las necesidades odontológicas de los pacientes para poder justificar la indispensable presencia de un odontólogo en la atención integral que debe ser prestada a estos pacientes. En este estudio se presentaron ciertas limitaciones como implementar carga viral y el recuento de CD4 dentro de las variables o utilizar posibles ayudas diagnosticas como las biopsias, frotis, radiografías y sialometrias para ofrecer diagnósticos más precisos.

Considerando todo lo anterior, podemos concluir que el estado de salud oral de este grupo de sujetos es inferior al de la población general colombiana al mostrar porcentajes superiores de la mayor parte de las patologías analizadas. Por tanto, se hace evidente la falta de asistencia odontológica y atención oportuna. Esto podría repercutir substancialmente en la aparición de alteraciones orales no relacionadas con el SIDA, conllevando así a altas necesidades de tratamiento odontológico. Se confirma de esta forma el derecho a la atención integral que este tipo de pacientes merece.

\section{AGRADECIMIENTOS}

Los autores agradecen a todos los participantes de este estudio, por su calidad humana y su gran interés por el desarrollo de esta investigación. 


\section{REFERENCIAS}

1. UN Joint Programme on HIV/AIDS. Global Report: UNAIDS report on the Global AIDS Epidemic: 2010. World Health Organization; 2010.

2. Departamento administrativo distrital de salud DADIS. Incidencia de las enfermedades de interés en salud pública en Cartagena (Colombia) 2004-2008. 2008.

3. Nittayananta W, Chanowanna N, Sripatanakul S, Winn T. Risk factors associated with oral lesions in HIV-infected heterosexual people and intravenous drug users in Thailand. Journal of Oral Pathology \& Medicine. 2001;30(4):224-30.

4. Dray-Spira R, Lert F. Marimoutou C, Bouhnik AD, Obadia Y. Socio-economic conditions. health status and employment among persons living with HIV/AIDS in France in 2001. AIDS Care. 2003;15(6):739-48.

5. Zungu-Dirwayi N, Shisana O, Louw J, Dana P. Social determinants for HIV prevalence among South African educators. AIDS Care. 2007;19(10):1296-303.

6. Santo AE, Tagliaferro EP, Ambrosano GM, Meneghim MC, Pereira AC. Dental status of Portuguese HIV+ patients and related variables: a multivariate analysis. Oral Dis. 2010;16(2):176-84

7. Pinzon E, Bravo S, Mendez F, Clavijo G, Leon M. Prevalencia y factores relacionados con la presencia de manifestaciones orales en pacientes con $\mathrm{VIH} /$ SIDA. Cali. Colombia. Colomb Med. 2008;39(4):34655.

8. Navas R, Santana Y, Morales T, Vargas E. Inequidades sociales: indicadores de salud-enfermedad bucal en pacientes VIH-SIDA. Multiciencias. 2009;9(3):313-19.

9. Noce CW, Ferreira SM, Silva Junior A, Dias EP. Association between socioeconomic status and HIVassociated oral lesions in Rio de Janeiro from 1997 to 2004. Pesqui Odontol Bras. 2009;23(2):149-54.

10. Valdespino JL, García-García ML, Conde-González CJ, Olaiz-Fernández G, Palma $\mathrm{O}$, Sepúlveda J. Prevalencia de infección por VIH en la población adulta en México: una epidemia en ascenso y expansión. Salud Publica Mex. 2007;49(3):386-94.

11. Lemos S, Oliveira F, Vencio E. Periodontal disease and oral hygiene benefits in HIV seropositive and AIDS patients. Medicina oral. patologia oral y cirugia bucal. 2010;15:417-21.

12. Santana Y. Navas R. Morón A. Chaparro N. García R. Suárez I. Condiciones de salud-enfermedad bucal en pacientes adultos VIH/SIDA. Acta Odontológica Venezolana. 2008;46(2). Disponible en: http:// www.actaodontologica.com/ediciones/2008/2/pdf/ condiciones_salud_enfermedad_bucal.pdf

13. Lambertini A, Prosperi A, Guerra ME, Tovar V, Brito F. Necesidades protésicas de los pacientes que acudieron al centro de atención a pacientes con enfermedades infectocontagiosas "Dra. Elsa la corte" (CAPEI) de la universidad Central de Venezuela entre noviembre del 2003 y noviembre del 2004. Acta odontol venez. 2006;44(3). Disponible en: http:// www.actaodontologica.com/ediciones/2006/3/ necesidades_protesicas.asp

14. Shiboski CH, Patton LL, Webster-Cyriaque JY, Greenspan D, Traboulsi RS, Ghannoum M, et al. The Oral HIV/AIDS Research Alliance: updated case definitions of oral disease endpoints. Journal of Oral Pathology \& Medicine. [Research Support. N.I.H.. Extramural]. 2009;38(6):481-8.

15. Ribeiro AdA, Portela M, Souza IPd, Relação entre biofilme. atividade de cárie e gengivite em crianças HIV+. Pesquisa Odontológica Brasileira. 2002;16:14450.

16. Ministerio de Salud de Colombia. III Estudio Nacionalde Salud Bucal ENSAB III. Bogota; 1998.

17. Hodgson TA, Greenspan D, Greenspan JS. Oral lesions of HIV disease and HAART in industrialized countries. Adv Dent Res. 2006;19(1):57-62.

18. Ramirez-Amador V, Anaya-Saavedra G, Calva JJ, Clemades-Perez-de-Corcho T, Lopez-Martinez C, Gonzalez-Ramirez I, et al. HIV-related oral lesions. demographic factors. clinical staging and antiretroviral use. Arch Med Res. 2006;37(5):646-54.

19. Nittayananta W, Chanowanna N, Jealae S, Nauntofte B, Stoltze K, Hyposalivation. xerostomia and oral health status of HIV-infected subjects in Thailand before HAART era. J Oral Pathol Med. 2010;39(1):2834.

20. Agbelusi GA, Wright AA. Oral lesions as indicators of HIV infection among routine dental patients in Lagos. Nigeria. Oral Diseases. 2005;11(6):370-3.

21. Patton LL, Phelan JA, Ramos-Gomez FJ, Nittayananta W, Shiboski $\mathrm{CH}$, Mbuguye TL. Prevalence and classification of HIV-associated oral lesions. Oral Diseases. [Review]. 2002;8 Suppl 2:98-109. 